\title{
Contemporary perceptions of human papillomavirus and penile cancer: Perspectives from a national survey
}

Michael E. Zavaski ${ }^{* 1}$; Julian Hanske ${ }^{* 1}$; Björn Löppenberg ${ }^{1}$; Alexander P. Cole ${ }^{1}$; Nawar Hanna; Soham Gupta ${ }^{2}$; Jairam R. Eswara; Mark A. Preston; Adam S. Kibel; Stuart R. Lipsitz; Maxine Sun ${ }^{1}$; Quoc-Dien Trinh ${ }^{1}$; Christian P. Meyer ${ }^{1,3}$

*Co-first authors

${ }^{1}$ Center for Surgery and Public Health and Division of Urologic Surgery, Brigham and Women's Hospital, Harvard Medical School, Boston, MA, United States; ${ }^{2}$ CURE Program, Dana-Faber Cancer Institute, Boston, MA, United States; ${ }^{3}$ Department of Urology, University Medical Center Hamburg-Eppendorf, Hamburg, Germany

Acknowledgments: Julian Hanske is supported by a grant from the Heinrich Hertz-Stiftung. Quoc-Dien Trinh is supported by an unrestricted educational grant from the Vattikuti Urology Institute and the Professor Walter Morris-Hale Distinguished Chair in Urologic Oncology at Brigham and Women's Hospital.

Cite as: Can Urol Assoc J 2018 July 31; Epub ahead of print. http://dx.doi.org/10.5489/cuaj.5366

Published online July 31, 2018

\section{$* * *$}

\section{Abstract}

Introduction: We aimed to assess the contemporary knowledge of human papillomavirus (HPV) and its association with penile cancer in a nationwide cohort from the US.

Methods: We used the Health Information National Trends Survey (HINTS), a crosssectional telephone survey performed in the U.S. initiated by the National Cancer Institute. The most recent iteration, HINTS 4 Cycle 4, was conducted in mail format between August 19 and November 17, 2014. Primary endpoints included knowledge of HPV and its causal relationship to penile cancer. Baseline characteristics included sex, age, education, race and ethnicity, income, residency, personal or family history of cancer, health insurance status, and internet use. Multivariable logistic regression assessed predictors of HPV and penile cancer knowledge.

Results: An unweighted sample of 3376 respondents was extracted from the HINTS 4, Cycle 4. Whereas $64.4 \%$ of respondents had heard of HPV, only $29.5 \%$ of these were aware that it could cause penile cancer. Men were significantly less likely to have heard 
of HPV than women (odds ratio [OR] 0.32; 95\% confidence interval [CI] 0.24-0.43). Older age; African-American, Asian, and “other race”; being married; from a lower education bracket; having a personal cancer history; and those without internet access were significantly less likely to have heard of HPV. None of our examined variables were independent predictors for the knowledge of the association of penile cancer and HPV. Conclusions: Our analysis of a large, nationally representative survey demonstrates that the majority of the American public is familiar with HPV, but lack a meaningful understanding between this virus and penile cancer. Primary care providers and specialists should be encouraged to intensify counselling about this significant association as a primary preventive measure of this potentially fatal disease.

\section{Introduction}

Penile cancer is a deadly yet rarely discussed genitourinary cancer in the United States. ${ }^{1}$ Globally, it accounts for up to $10 \%$ of reported cancers in the developing world, ${ }^{2}$ presumably from certain risk factors of penile cancer such as chronic inflammation from poor access to sanitation and persistent human papillomavirus (HPV) infection. ${ }^{3}$ In the developed world, penile cancer is less common yet still affects a large number of men with HPV being the major risk factor. In the US, HPV is estimated to cause up to 1000 new penile cancer cases annually. ${ }^{4}$

Genital HPV is a highly prevalent sexually transmitted virus. While most infections are self-limited, host and viral factors may combine to cause persistent infections which can lead to cancer. The virus is responsible for many vulvar, vaginal, anal, oropharyngeal and penile cancers. But by far the best-known example is cervical cancer. Large systematic surveys have demonstrated widespread awareness of the HPVcervical cancer relationship - especially among women. ${ }^{5}$ Yet despite high rates of HPV prevalence and transmission between both sexes, less is known about public awareness of HPV's effects on men's health. Recent studies demonstrated that men had inadequate knowledge about the benefits of HPV vaccination and consequently had insufficient interest in pursuing the vaccine. ${ }^{6,7}$ Other studies show that HPV knowledge is increasing and understanding of the disease is patterned by sex, education, age and other sociodemographic factors consistent with the Knowledge Gap Hypothesis. ${ }^{5}$ This hypothesis observes the flow of information on a given topic can lead to differential learning among members of certain groups, impacted by such things as race, ethnicity or socioeconomic status. In this study, we used a nationally representative survey to investigate public knowledge of HPV and its relationship to penile cancer. To our knowledge, this is the first comprehensive population-level study assessing public awareness of this important concept. 


\section{Methods}

\section{Data source}

The Health Information National Trends Survey (HINTS) is a cross-sectional telephone survey of the US. HINTS was initiated in 2003 and has provided updates on changing patterns, needs, and information opportunities in health care. Initiated by the National Cancer Institute, HINTS data collection program has monitored changes in health conformation seeking behavior and health perception in four consecutive surveys thus far. The most recent iteration, HINTS 4 Cycle 4, was conducted in mail format between August 19 to November 17, 2014. An equal-probability sample of addresses was selected from sampling strata and the second stage of sampling consisted of a within-household sample selection. Only completed questionnaires were considered eligible for inclusion that resulted in 3,677 participants. The overall response rate was 34.44\%. A recent analysis found that HINTS estimates could be biased toward higher health information seeking individuals, however these can be accounted for using standard weighting procedures, which incorporate calibration adjustments using data from the American Community Survey and the National Health Interview survey. ${ }^{8}$

Outcome measures

We examined the perception and knowledge of HPV infection and its association with penile cancer as endpoints of interest. Survey questions encompassed the following content: Have you ever heard of HPV? HPV stands for Human Papillomavirus. It is not $H I V, H S V$, or herpes. (yes/no); Do you think HPV can cause penile cancer? (yes/no/unsure); Do you think HPV is a sexually transmitted disease (STD)? (yes/no/unsure); Do you think HPV requires medical treatment or will it usually go away on its own without treatment? (requires medical treatment/will usually go away on its own.

Independent variables

Sociodemographic variables included in the analysis were sex, age, education, race/ethnicity, income, residency, personal or family history of cancer, health insurance status, and internet use. All independent variables were dichotomized or recoded as indicated.

\section{Statistical analyses}

Descriptive statistics focused on frequencies and proportions for categorical variables. The chi-square test was used to assess the statistical significance of medians and proportions, respectively. Multivariable logistic regression analyzes assessed predictors of HPV and penile cancer knowledge and perceptions. The cohort was stratified by gender and results were weighted using the jackknife method with replicate weights to 
reflect the US population based on the complex survey design. All analyses were performed using SAS, Version 9.3. A 2-sided p-value of less than 0.05 was used as the threshold for statistical significance. The study received a waiver by the institutional review board.

\section{Results}

Study cohort

After weighting, we identified an overall sample of 222.8 million participants $\left(\mathrm{n}_{\text {unweighted }}=3,376\right)$ within HINTS 4, Cycle 4. This resulted in a proportion of approximately 109.0 million men $\left(\mathrm{n}_{\text {unweighted }}=1,370 ; 48.9 \%\right)$ and 113.7 million women $\left(\mathrm{n}_{\text {unweighted }}=2,006 ; 51.1 \%\right)$. Most were younger than 65 years old (82.5\%) non-Hispanic Whites (66.6\%) and married (53.7\%). The rate of insured individuals was $87.3 \%, 8.5 \%$ indicated having a personal history of cancer, and 70.9\% reported a family history of cancer (Table 1).

Respondent knowledge and awareness related to HPV

Although $64.4 \%$ of the participants had knowledge of HPV, the majority was unsure (55.2\%) if HPV can cause penile cancer. 29.5\% answered that they knew about the link between penile cancer and HPV and 15.3\% answered 'no' to this question. (Table 2).

Identification of predictors through survey questions

Men were less likely to have ever heard of HPV than women (odds ratio [OR]: 0.32, 95\% confidence interval [CI]: 0.24-0.43). Furthermore, participants were less likely informed if they were older than 65 years old (OR: 0.38, 95\%CI: 0.27-0.53), African-American (OR: 0.46, 95\%CI: 0.27-0.78), Asian (OR: 0.25, 95\%CI: 0.11-0.55), “other” race (OR: 0.31, 95\%CI: 0.12-0.84), married (OR: 0.59, 95\%CI: 0.43-0.81), or coming from a lower education bracket (some high school: OR: 0.38, 95\%CI: 0.17-0.85) having a personal cancer history (OR: 0.65, 95\%CI: 0.44-0.95), and lacking Internet access (OR: 0.59, 95\%CI: 0.40- 0.87). Having children (OR: 1.86, 95\%CI: 1.22-2.82), higher income (\$>75,000: OR: 1.83, 95\%CI: 1.08-3.11), urban residency (OR: 1.85, 95\%CI: 1.14-3.00), and reported family cancer history (OR: 1.85, 95\%CI: 1.28-2.67) predisposed participants to HPV knowledge (Table 3).

No variables examined were associated with knowledge of a link between HPV and penile cancer (Table 4).

\section{Discussion}

Most sexually active young adults will acquire genital HPV at some point. ${ }^{9,10}$ It ranks atop sexually transmitted infections in the US and is a key preventable risk factor among numerous types of cancer-nearly all cervical cancers as well as many vulvar, vaginal, anal, oropharyngeal and the vast majority of cases of penile cancers. ${ }^{1,11}$ While recent 
strides in awareness of HPV's relationship with cervical cancer have been made - public knowledge about its relationship to penile cancer remains limited. ${ }^{12,13}$ In our current study, we confirm a general lack of awareness regarding the association between HPV and penile cancer among a nationally representative sample of men and women.

A similar knowledge gap was once seen in cervical cancer. Less than $1 \%$ of respondents in a CDC focus groups convened in 2003 were even aware of an association. ${ }^{14}$ However, much has changed since that time. The first HPV vaccine approved to prevent cervical cancer was approved in the US in 2006. Professional guidelines and widespread public campaigns soon followed. ${ }^{15,16}$ As a result, awareness of the causal relationship between HPV and cervical cancer increased: A population-based survey from 2013 using HINTS showed that two thirds of respondents (including both genders and a large variety of social backgrounds) knew that HPV causes cervical cancer. ${ }^{5}$ In some populations this is even higher: a survey of college women in 2008 revealed that over $85 \%$ knew that HPV causes cervical cancer. ${ }^{17}$ Although there are no studies showing an association of HPV knowledge and lower disease incidence (e.g. HPV infection or cervical cancer), recent meta-analyses have shown a substantial benefit of education programs on cervical cancer screening rates. ${ }^{18}$ While these improvements are laudable and may benefit thousands of women at risk of cervical cancer, similar strides in awareness of HPV's effects on men's health have not been seen. In our nationally representative survey, only a small minority of American men and women are aware of the link between HPV and penile cancer.

Prior small studies have shown similar knowledge deficits. In 2000, a survey of 322 incoming students at a New England university assessed knowledge of HPV and its relationship to penile dysplasia of which $13.4 \%$ of respondents correctly reported an association with no significant differences between men and women. ${ }^{19}$ More recently in a 2014 study of 400 adult Chinese men having sex with men, only 34.7\% perceived HPV infection would result in high or very high chance of causing penile or anal cancer. But even among these men at high risk for the virus, little was known of the potential mortality of penile cancer and only about one third expressed a high or very high degree of fear towards penile or anal cancer. ${ }^{20}$

It is possible that the low incidence of penile cancer may account for the lack of widespread knowledge about HPV's key causal role. But this explanation is not satisfying. In our study, the older men most at risk of penile cancer were also those least likely to be aware of it. ${ }^{2}$ And women (who obviously are at no risk of penile cancer) have a better understanding of HPV as a disease entity ${ }^{19,21}$ Thus, factors like education and exposure to health education materials may be more impactful than the actual risk of getting the disease. While disheartening that so few men are aware of the risk of HPV, the finding of improved knowledge in women (who have no risk of penile cancer but are probably more inclined to receive information about cervical cancer and HPV) does 
suggest that targeted interventions to improve public knowledge may substantially increase understanding.

Women's health advocates and physicians have long advocated the benefits of HPV vaccination. The practice of vaccinating adolescent men to prevent HPV-associated cancers is also increasing. ${ }^{22}$ Alongside a reduction in cervical, vulvar vaginal cancers for female sexual partners, protection from some anal, oro-pharyngeal and penile cancers constitutes a direct benefit for men being vaccinated. ${ }^{23}$ As this benefit becomes more publicized, more and more men may seek out vaccinations and gain protection from these deadly cancers. At present, vaccination coverage goals outlined by the CDC are substantially below goal for both women and men with $37.6 \%$ of adolescent girls and $13.9 \%$ of adolescent boys having received the 3 -dose series for HPV vaccination. ${ }^{24}$ In order to achieve widespread immunization, spreading knowledge of these benefits, and assessing changes in knowledge will be key.

While knowledge and medical education appear to be an important part of successful vaccination campaigns, system-level barriers seem to be equally important. Interestingly, data from the US have paradoxically shown that underserved populations have higher HPV vaccination rates. ${ }^{25}$ Moreover, there are concerns that geographical location from clinic/hospital may impact the quality of care received - for example, rural patients tend to be less likely to receive adequate care, including HPV vaccination,

possibly due to impaired access to the health care system. ${ }^{26,27}$ Such considerations may be of special interest to large countries such as Canada.

The strengths of our study include its broad scope and the ability of HINTS to survey a broadly representative national population. Limitations include the study's cross-sectional nature and the fact that the HINTS study questionnaire investigates access and need for health information rather than exploring overall understanding of STDs and cancer knowledge. Moreover, the HINTS response rate was low at just above 30\% which may limit generalizability, although the survey did take into account modality coverage and sampling in an attempt to limit these biases. ${ }^{28}$ Future studies which assess changes in knowledge over time would allow for more nuanced assessments of the factors involved in disseminating knowledge of penile cancer and HPV and therefore allow even more targeted interventions to increase awareness and reduce men's risk for these largely preventable cancers.

\section{Conclusion}

In this nationally representative study, the majority of respondents demonstrated knowledge of HPV as an STD however there remained a significant deficit in knowledge of its association with penile cancer. Even among the subset of men and women familiar with HPV, only $29.5 \%$ suspected a causal relationship between HPV and penile cancer. Given the potential for preventative strategies to dramatically reduce the major 
modifiable risk factor of this deadly cancer, there is a great need to educate US men about this disease. The example of cervical cancer shows that increasing awareness is achievable - it will be up to physicians, health educators and public health officials to achieve this goal and help thwart this largely preventable male cancer.

\section{References:}

1. Christodoulidou M, Sahdev V, Houssein S, et al. Epidemiology of penile cancer. Current problems in cancer 2015;39:126-36.

2. Bleeker MC, Heideman DA, Snijders PJ, et al. Penile cancer: epidemiology, pathogenesis and prevention. World journal of urology 2009;27:141-50.

3. Daling JR, Madeleine MM, Johnson LG, et al. Penile cancer: importance of circumcision, human papillomavirus and smoking in in situ and invasive disease. International journal of cancer Journal international du cancer 2005;116:606-16.

4. Pow-Sang MR, Ferreira U, Pow-Sang JM, et al. Epidemiology and natural history of penile cancer. Urology 2010;76:S2-6.

5. Blake KD, Ottenbacher AJ, Finney Rutten LJ, et al. Predictors of human papillomavirus awareness and knowledge in 2013: gaps and opportunities for targeted communication strategies. American journal of preventive medicine 2015;48:402-10.

6. Gerend MA, Barley J. Human papillomavirus vaccine acceptability among young adult men. Sexually transmitted diseases 2009;36:58-62.

7. Reiter PL, Brewer NT, Smith JS. Human papillomavirus knowledge and vaccine acceptability among a national sample of heterosexual men. Sexually transmitted infections 2010;86:241-6.

8. Maitland A, Lin A, Cantor D, et al. A Nonresponse Bias Analysis of the Health Information National Trends Survey (HINTS). Journal of Health Communication 2017;22:545-53.

9. Satterwhite CL, Torrone E, Meites E, et al. Sexually transmitted infections among US women and men: prevalence and incidence estimates, 2008. Sexually transmitted diseases 2013;40:187-93.

10. McQuillan G, Kruszon-Moran D, Markowitz LE, et al. Prevalence of HPV in Adults Aged 18-69: United States, 2011-2014. NCHS data brief 2017:1-8.

11. Castellsague X, Bosch FX, Munoz N, et al. Male circumcision, penile human papillomavirus infection, and cervical cancer in female partners. The New England journal of medicine 2002;346:1105-12.

12. Mohammed KA, Subramaniam DS, Geneus CJ, et al. Rural-urban differences in human papillomavirus knowledge and awareness among US adults. Preventive medicine 2018;109:39-43. 
13. McBride KR, Singh S. Predictors of Adults' Knowledge and Awareness of HPV, HPV-Associated Cancers, and the HPV Vaccine: Implications for Health Education. Health education \& behavior : the official publication of the Society for Public Health Education 2018;45:68-76.

14. Friedman AL, Shepeard H. Exploring the knowledge, attitudes, beliefs, and communication preferences of the general public regarding HPV: findings from CDC focus group research and implications for practice. Health education \& behavior : the official publication of the Society for Public Health Education 2007;34:471-85.

15. Ylitalo KR, Lee H, Mehta NK. Health care provider recommendation, human papillomavirus vaccination, and race/ethnicity in the US National Immunization Survey. American journal of public health 2013;103:164-9.

16. Saslow D, Castle PE, Cox JT, et al. American Cancer Society Guideline for human papillomavirus (HPV) vaccine use to prevent cervical cancer and its precursors. CA: a cancer journal for clinicians 2007;57:7-28.

17. Sandfort JR, Pleasant A. Knowledge, attitudes, and informational behaviors of college students in regard to the human papillomavirus. Journal of American college health : J of ACH 2009;58:141-9.

18. Musa J, Achenbach CJ, O’Dwyer LC, et al. Effect of cervical cancer education and provider recommendation for screening on screening rates: A systematic review and meta-analysis. PLoS ONE 2017;12:e0183924.

19. Baer H, Allen S, Braun L. Knowledge of human papillomavirus infection among young adult men and women: implications for health education and research. Journal of community health 2000;25:67-78.

20. Lau JT, Wang Z, Lau M, et al. Perceptions of HPV, genital warts, and penile/anal cancer and high-risk sexual behaviors among men who have sex with men in Hong Kong. Archives of sexual behavior 2014;43:789-800.

21. Gerend MA, Magloire ZF. Awareness, knowledge, and beliefs about human papillomavirus in a racially diverse sample of young adults. The Journal of adolescent health : official publication of the Society for Adolescent Medicine 2008;42:237-42.

22. Elam-Evans LD, Yankey D, Jeyarajah J, et al. National, regional, state, and selected local area vaccination coverage among adolescents aged 13-17 years-United States, 2013. MMWR Morbidity and mortality weekly report 2014;63:62533.

23. Harper DM. Prevention of human papillomavirus infections and associated diseases by vaccination: a new hope for global public health. Public health genomics 2009;12:319-30. 
24. Schwartz JL, Easterling LA. State Vaccination Requirements for HPV and Other Vaccines for Adolescents, 1990-2015. Jama 2015;314:185-6.

25. Holman DM, Benard V, Roland KB, et al. Barriers to Human Papillomavirus Vaccination Among US Adolescents: A Systematic Review of the Literature. JAMA pediatrics 2014;168:76-82.

26. Henry KA, Swiecki-Sikora AL, Stroup AM, et al. Area-based socioeconomic factors and Human Papillomavirus (HPV) vaccination among teen boys in the United States. BMC public health 2017;18:19.

27. Vielot NA, Butler AM, Brookhart MA, et al. Patterns of Use of Human Papillomavirus and Other Adolescent Vaccines in the United States. The Journal of adolescent health : official publication of the Society for Adolescent Medicine 2017;61:281-87.

28. Westat. Health Information National Trends Survey 4 (HINTS 4) Cycle 2 Methodology Report. 2013:46. 
Figures and Tables

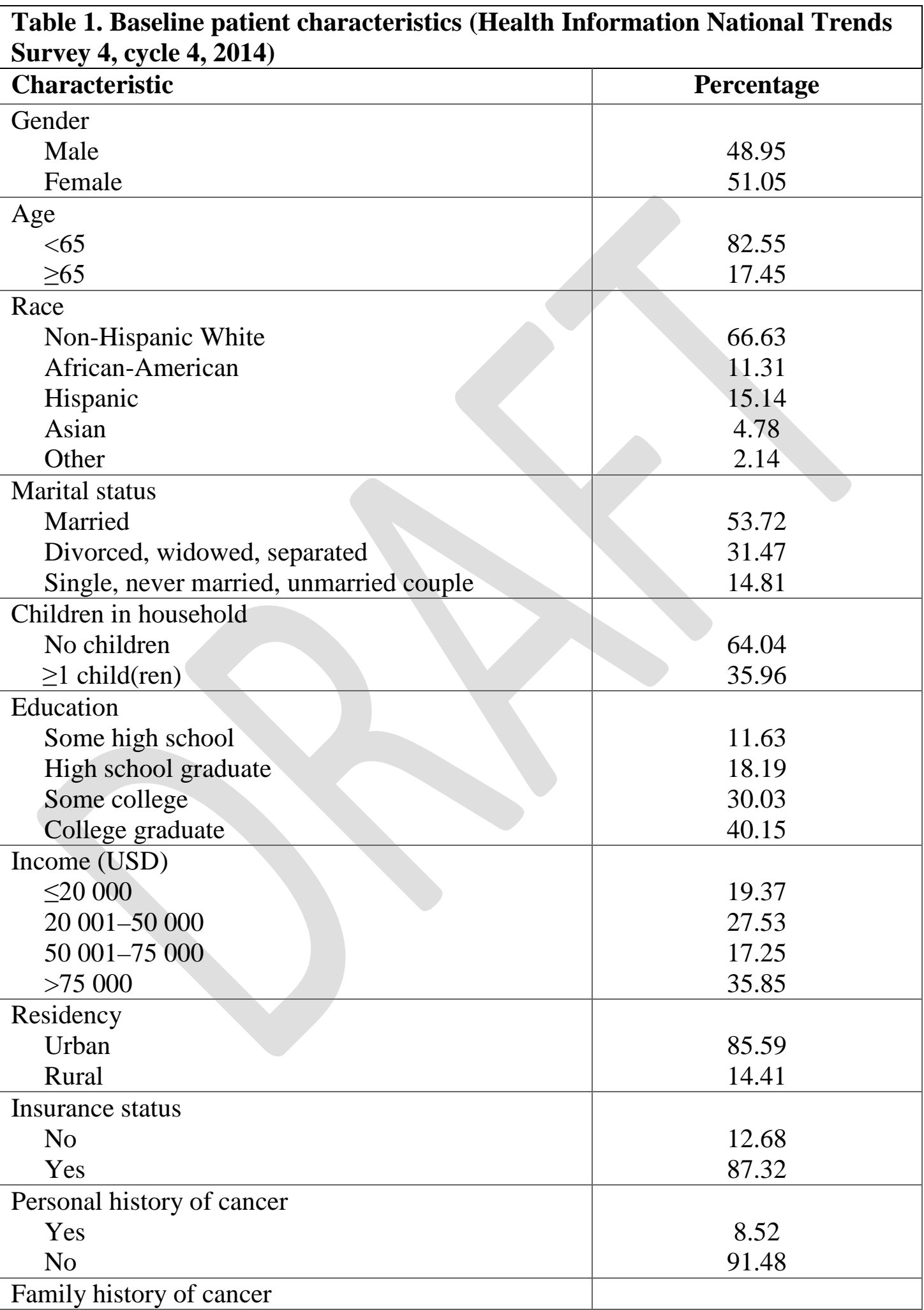




\begin{tabular}{|c|c|}
\hline Yes & 70.94 \\
No & 29.06 \\
\hline
\end{tabular}

Tabulated variables are from weighted estimates.

Table 2. Univariate responses to HPV questions/simple proportions (Health Information National Trends Survey 4, cycle 4, 2014)

\begin{tabular}{|l|c|c|c|}
\hline Survey question & \multicolumn{3}{|c|}{ Percentage } \\
\hline Have you ever heard of HPV? & No & Yes & Not sure \\
\hline Do you think HPV can cause penile cancer? & 35.65 & 64.35 & - \\
\hline Do you think HPV can cause cervical cancer? & 15.34 & 29.47 & 55.18 \\
\hline Do you think HPV is a STD? & 0.97 & 77.99 & 21.04 \\
\hline Do you think HPV needs medical treatment? & 30.15 & 69.85 & - \\
\hline
\end{tabular}

Tabulated variables are from weighted estimates. HPV: human papillomavirus; STD: sexually transmitted disease. 


\begin{tabular}{|c|c|c|}
\hline Variable & OR (95\% CI) & $\mathbf{p}$ \\
\hline $\begin{array}{l}\text { Gender } \\
\text { Male } \\
\text { Female }\end{array}$ & $\begin{array}{c}0.32 \text { (0.24-0.43) } \\
\text { Ref. }\end{array}$ & $\begin{array}{c}<0.001 \\
-\end{array}$ \\
\hline $\begin{array}{l}\text { Age } \\
\quad<65 \\
\quad \geq 65\end{array}$ & $\begin{array}{c}\text { Ref. } \\
\mathbf{0 . 3 8}(\mathbf{0 . 2 7}-\mathbf{0 . 5 3 )})\end{array}$ & $\begin{array}{c}- \\
<0.001\end{array}$ \\
\hline $\begin{array}{l}\text { Race } \\
\text { Non-Hispanic White } \\
\text { African American } \\
\text { Hispanic } \\
\text { Asian } \\
\text { Other }\end{array}$ & $\begin{array}{c}\text { Ref. } \\
\mathbf{0 . 4 6}(\mathbf{0 . 2 7}-\mathbf{0 . 7 8 )} \\
0.75(0.45-1.26) \\
\mathbf{0 . 2 5}(\mathbf{0 . 1 1}-\mathbf{0 . 5 5}) \\
\mathbf{0 . 3 1}(\mathbf{0 . 1 2}-\mathbf{0 . 8 4})\end{array}$ & $\begin{array}{c}- \\
\mathbf{0 . 0 0 4} \\
0.278 \\
<\mathbf{0 . 0 0 1} \\
\mathbf{0 . 0 2 2}\end{array}$ \\
\hline $\begin{array}{l}\text { Marital status } \\
\text { Married } \\
\text { Divorced, widowed, separated } \\
\text { Single, never married, unmarried } \\
\text { couple }\end{array}$ & $\begin{array}{l}\mathbf{0 . 5 9}(\mathbf{0 . 4 3 - 0 . 8 1 )} \\
1.06(0.71-1.58) \\
\text { Ref. }\end{array}$ & $\begin{array}{c}\mathbf{0 . 0 0 1} \\
0.772 \\
-\end{array}$ \\
\hline $\begin{array}{l}\text { Children in household } \\
\text { No children } \\
\geq 1 \text { child(ren) }\end{array}$ & $\begin{array}{l}\text { Ref. } \\
1.86(1.22-2.82)\end{array}$ & $\stackrel{-}{0.004}$ \\
\hline $\begin{array}{l}\text { Education } \\
\text { Some high school } \\
\text { High school grad } \\
\text { Some college } \\
\text { College grad }\end{array}$ & 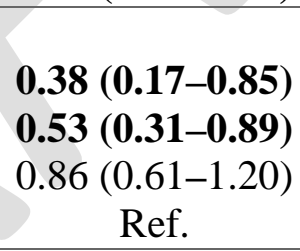 & $\begin{array}{c}\mathbf{0 . 0 1 9} \\
\mathbf{0 . 0 1 7} \\
0.365 \\
-\end{array}$ \\
\hline $\begin{array}{l}\text { Income (USD) } \\
\quad \leq 20000 \\
20001-50000 \\
50001-75000 \\
>75000\end{array}$ & $\begin{array}{c}\text { Ref. } \\
1.19(0.70-2.04) \\
\mathbf{1 . 9 8}(\mathbf{1 . 0 3}-3.79) \\
\mathbf{1 . 8 3}(\mathbf{1 . 0 8}-\mathbf{3 . 1 1})\end{array}$ & $\begin{array}{l}- \\
0.519 \\
\mathbf{0 . 0 4 0} \\
\mathbf{0 . 0 2 5}\end{array}$ \\
\hline $\begin{array}{l}\text { Residency } \\
\text { Urban } \\
\text { Rural }\end{array}$ & $\begin{array}{c}1.85 \text { (1.14-3.00) } \\
\text { Ref. }\end{array}$ & $\begin{array}{c}0.013 \\
-\end{array}$ \\
\hline $\begin{array}{l}\text { Insurance status } \\
\text { No } \\
\text { Yes }\end{array}$ & $\begin{array}{c}0.79(0.49-1.30) \\
\text { Ref. }\end{array}$ & $\begin{array}{c}0.360 \\
-\end{array}$ \\
\hline $\begin{array}{l}\text { Personal cancer history } \\
\text { Yes } \\
\text { No }\end{array}$ & $\begin{array}{c}0.65(0.44-0.95) \\
\text { Ref. }\end{array}$ & $\begin{array}{c}0.025 \\
-\end{array}$ \\
\hline Family cancer history & & \\
\hline
\end{tabular}




\begin{tabular}{|l|c|c|}
\hline $\begin{array}{l}\text { Yes } \\
\text { No }\end{array}$ & $\mathbf{1 . 8 5 ( 1 . 2 8 - 2 . 6 7 )}$ & $\mathbf{0 . 0 0 1}$ \\
\hline $\begin{array}{l}\text { Do you ever go online to access the } \\
\text { internet or to send/receive email? } \\
\text { Yes }\end{array}$ & Ref. & \\
No & Ref. & - \\
\hline
\end{tabular}

Tabulated variables are from weighted estimates. CI: confidence interval; HPV: human papillomavirus; OR: odds ratio. 


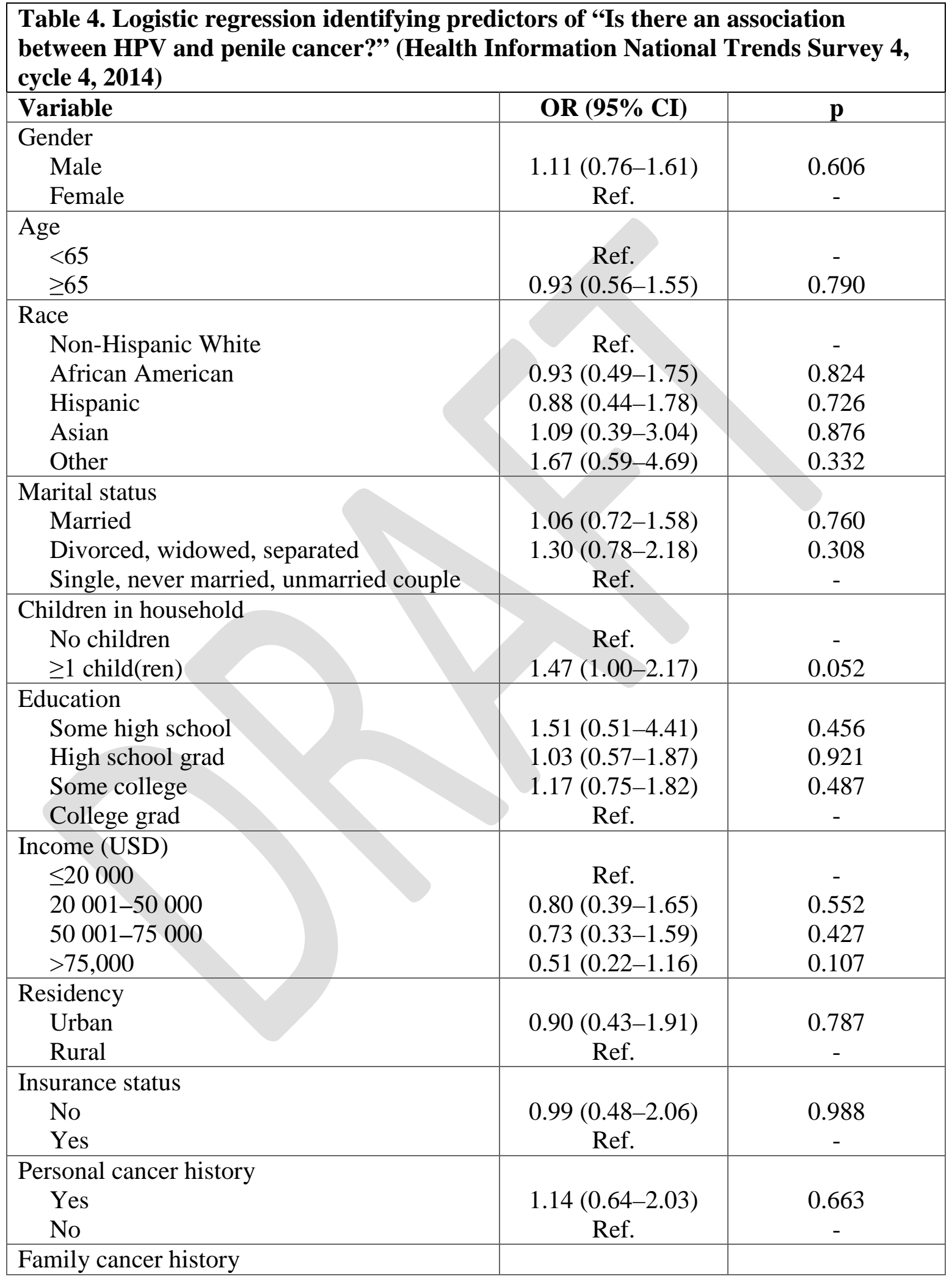




\begin{tabular}{|l|c|c|}
\hline \multicolumn{1}{|l|}{$\begin{array}{c}\text { Yes } \\
\text { No }\end{array}$} & $0.91(0.62-1.35)$ & 0.651 \\
\hline $\begin{array}{l}\text { Do you ever go online to access the internet } \\
\text { or to send/receive email? } \\
\begin{array}{l}\text { Yes } \\
\text { No }\end{array}\end{array}$ & Ref. \\
\hline
\end{tabular}

Tabulated variables are from weighted estimates. CI: confidence interval; HPV: human papillomavirus; OR: odds ratio. 